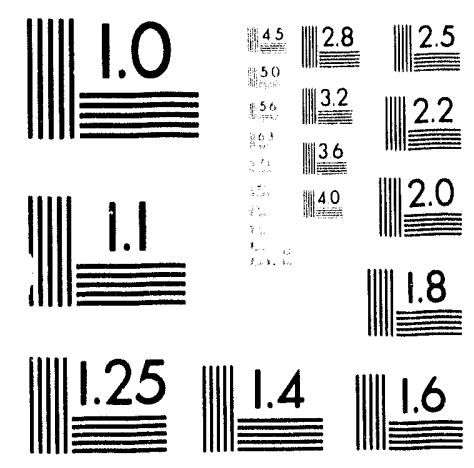



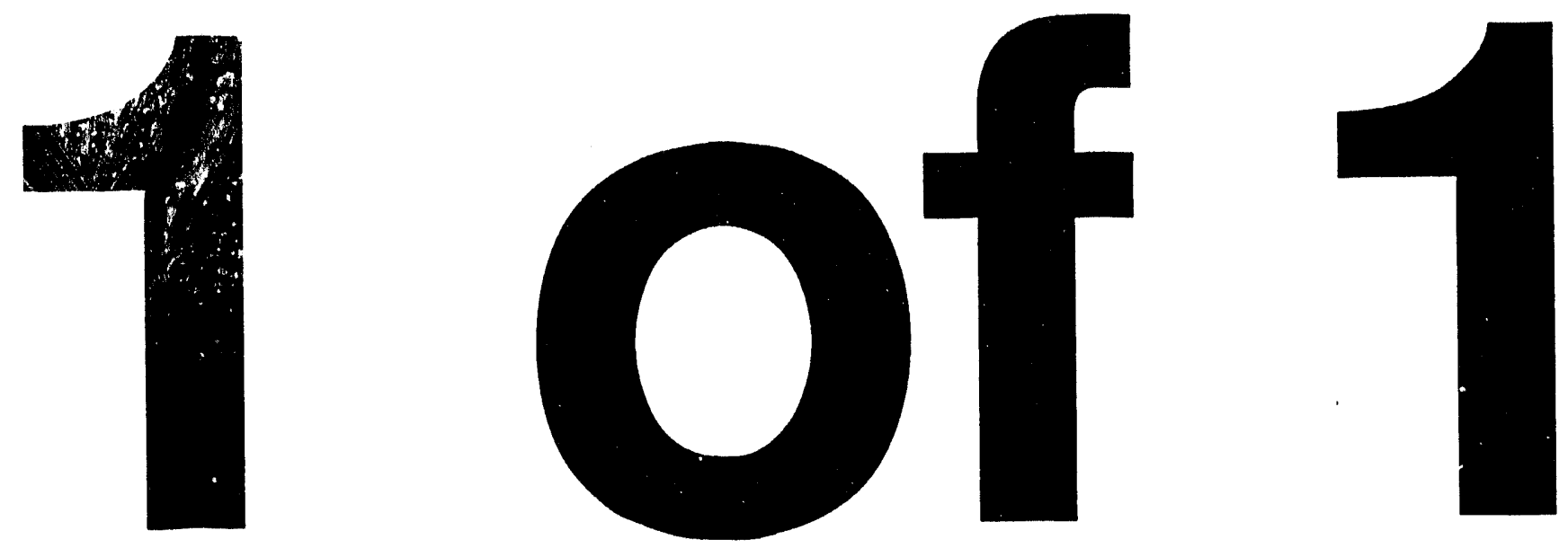


\title{
Simulation of Foam Displacement in Porous Media
}

\author{
A. R. Kovscek, ${ }^{*}$ T.W. Patzek, ${ }^{\dagger}$ and C. J. Radke* \\ *Department of Chemical Engineering \\ University of California \\ ${ }^{\dagger}$ Department of Materials Science and Mineral Engineering \\ University of California \\ and \\ Earth Sciences Division \\ Lawrence Berkeley Laboratory \\ University of California \\ Berkeley, California 94720
}

August 1993

This work was supported by the Assistant Secretary for Fossil Energy, Office of Oil, Gas and Shale Technologies, of the U.S. Department of Energy under Contract No. DE-AC03-76SF00098. 


\begin{abstract}
Foam is an excellent fluid for achieving mobility control of gas in porous media. Practical application of foams for EOR processes, however requires a predictive model of foam displacement. Further, quantitative information on foam-flow behavior at reservoir flow rates and pressures is required as input to any field-scale modeling.

An experimental and mechanistic-modeling study is reported for the transient flow of foam through 1.3 $\mu \mathrm{m}^{2}(1.3 \mathrm{D})$ Boise sandstone at backpressures in excess of $5 \mathrm{MPa}$ ( $700 \mathrm{psi}$ ) over a quality range from 0.80 to 0.99 . Total superficial velocities range from as little as 0.42 to $2.20 \mathrm{~m} /$ day ( $1.4 \mathrm{ft} /$ day to $7 \mathrm{ft} /$ day). Sequential pressure taps and gamma-ray densitometry measure flow resistance and in-situ liquid saturations, respectively. We garner experimental pressure and saturation profiles in both the transient and steady states.

Adoption of a mean-size foam-bubble conservation equation along with the traditional reservoir simulation equations allows mechanistic foam simulation. Since foam mobility depends heavily upon its texture, the bubble population balance is both useful and necessary as the role of foam texture must be incorporated into any model which seeks accurate prediction of flow properties. Our model employs capillary-pressure-dependent kinetic expressions for lamellae generation and coalescence and also a term for trapping of lamellae.
\end{abstract}

References and illustrations at end of paper
Additionally, the effects of surfactant chemical transport are included.

We find quantitative agreement between experimental and theoretical saturation and pressure profiles in both the transient and steady states.

\section{Introduction}

Foam is useful for controlling mobility of gases in porous media. Foam is relatively cost effective because it is mainly gas with stabilization of the gas/liquid interface provided by a relatively low concentration of surfactant (of order $1 \mathrm{wt} \%$ ) within the aqueous phase. Since the gaseous portion of foam is dispersed, gas-phase flow mobility is greatly reduced and hence gravity override and viscous fingering through high-permeability streaks may be reduced. However, practical implementation of foams for mobility control in enhanced oil recovery (EOR) processes has been hindered because a general understanding and a predictive model of foam flow does not currently exist.

Most previous studies were Eddisionian and focused upon the steady state. Although transient flow (i.e., displacement) is the most relevant to EOR, a reliable experimental data set that includes transient pressure and in-situ saturation profiles (along the length of a core) does not exist for foam flow. The most notable attempts at modeling foam flow have focused either on predicting transient flow ${ }^{1}$ or on predicting steady state results 2,3 , but not both. Additionally, the transient experiments of Friedmann 
et al ${ }^{1}$ were for gas frontal advance rates between roughly 10 and $1000 \mathrm{~m} /$ day.

In recognition oi the above issues, we undertook a simultaneous experimental and simulation study of transient foam displacement. Here we demonstrate the usefulness and generality of the population-balance approach ${ }^{4}$ for simulating transient and steady state foam flow in porous media.

The propagation of foam fronts within Boise sandstone at low displacement rates are tracked experimentally under a variety of injection modes and initial conditions. Specifically, three different types of foam displacement are considered: (1) simultaneous injection of gas and surfactant solution at constant mass injection rates into a core completely saturated with surfactant solution, (2) simultaneous injection of gas and surfactant solution into a brine-filled core again at constant mass injection rates, and (3) gas injection into a surfactant saturated core at a fixed injection and exit pressures. Total superficial velocities in the transient mode are generally $1 \mathrm{~m} /$ day ( $3 \mathrm{ft}$ day) or less. Under steady-state conditions, the liquid flow rate is varied while holding the gas flow rate constant (and vice versa) and measuring the resulting pressure-drop behavior. We concentrate on oil-free systems to avoid confusing foam propagation with foam/oil interaction.

\section{Pore-Level Schematic of Foam Flow}

Gillis and Radke ${ }^{5}$ proposed Fig. 1 as a summary of the pore-level distribution of foam. In this highly schematic picture, cross-hatched circles refer to waterwet sand grains. Wetting surfactant solution is denoted as the dotted phase. Foam bubbles are either unshaded or darkly shaded, depending upon whether they are stationary or flowing. For illustrative purposes only, the largest pore channels lie at the top of the figure while the smallest lie at the bottom.

In compliance with strong capillary forces, wetting liquid occupies the smallest pore space and clings to the surface of sand grains as wetting films. The aqueous wetting phase maintains continuity throughout the pore structure shown in Fig. 1 so that the aqueous-phase relative permeability function is unchanged in the presence of foam 6-11. Minimal amounts of liquid transport as lamellae. Unshaded flowing foam transports as trains of bubbles through the largest and least resistive flow channels. Because the smallest pore channels are occupied solely by wetting liquid and the largest pore channels carry flowing foam, bubble trapping occurs in the intermediate-sized pores.

Foam reduces gas mobility in two ways. First, stationary or trapped foam blocks a large number of channels that otherwise carry gas. Gas tracer studies 1,5 measure the fraction of gas trapped within a foam at steady state in sandstones to lie between 85 and $99 \%$. Second, bubble trains within the flowing fraction encounter drag because of the presence of pore walls and constrictions 12 , and because the gas/liquid interfacial area of a flowing foam bubble is constantly being rearranged by viscous and capillary forces 13 .

These trains are in a constant state of rearrangement. Foam texture arises through a balance between varied and complicated foam generation and destruction mechanisms. Regardless of whether foam bubbles are generated in situ or externally, the are molded and shaped by the porous medium 3,14 . Bubbles and lamella transport some distance, are destroyed, and then reformed. Further, trains halt when the local pressure gradient is insufficient to keep them mobilized, and other trains then begin to flow. No single bubble or train is conserved over any large distance (i.e., the length of several pore bodies). Bubble trains exist only on a time averaged sense. More thorough reviews of foam generation, coalescence, and transport on the pore level are given in refs. 14 and 15 .

\section{Experimental Apparatus and Procedures}

Persoff et al. ${ }^{16}$ originally designed the apparatus used for the experimental foam floods. More detail is also located in refs. 15 and 17 . The centerpiece of the apparatus is a vertically mounted, $60-\mathrm{cm}$ long, 5.1$\mathrm{cm}$ diameter, $1.3-\mu \mathrm{m}^{2}$ Boise sandstone core with a porosity of 0.25 . The core is epoxy-mounted into a 316 stainless steel sleeve designed to withstand pressures up to $20 \mathrm{MPa}$ (3000 psi). A Mity-mite dome-loaded backpressure regulator (Grove Valve and Regulator Company, Emeryville, CA) maintains core backpressure.

Nitrogen gas and foamer solution are injected at the top of the core to prevent buoyancy-driven gas flow. For injection at fixed mass flow rates, a Brooks 5850C mass flow controller (Emerson Electric, Hatfield, PA) meters nitrogen flow yielding gas Darcy velocities from 0.30 to $2.1 \mathrm{~m} /$ day (1 to $7 \mathrm{ft} /$ day) at 5 $\mathrm{MPa}$ (700 psi) backpressure. An ISCO 500D syringe pump (Instrumentation Specialties Company, Lincoln, NE) provides liquid flow. Liquid velocities as low as $0.009 \mathrm{~m} /$ day $(0.03 \mathrm{ft} /$ day $)$ are employed.

Liquid saturation profiles are measured by scanning gamma-ray densitometry utilizing a $47 \mathrm{mCi}$ Cs-137 source. After a simple calibration where gamma-ray beam intensity (counts/s falling within a $662 \mathrm{keV}$ peak) is measured at preselected points along the core at $0 \%\left(I_{d}\right)$ and $100 \%$ liquid saturation $\left(I_{W}\right)$. the liquid content at any previously calibrated point in the core is found from the Beer-Lambert law, $S_{W}=$ $\left[\ln \left(I_{d} / I\right)\right] /\left[\ln \left(I_{d}-I_{W}\right)\right]$, where $I$ is the intensity measured at any unknown saturation. Mounting of the gamma-ray source and detector on a translating carriage allows the core to be scanned. 
Pressure taps are located at the core inlet, outlet, and at $10 \mathrm{~cm}(4 \mathrm{in}$.$) intervals along the core, and are$ sealed with Swagelok O-seal (Crawford Fitting Company, Solon, Ohio) fittings. Pressure is measured using a single Paroscientific $43 \mathrm{KT}$ piezoelectric quartz-crystal pressure transducer (Paroscientific, Redmond, 'WA). A Scannivalve 12L7 multiplexing valve (Scannivalve, San Diego, CA) allows all pressure taps to be visited sequentially and rapidly. An HP-9000 (Hewlett Packard Co., Mountain View, CA) controls the apparatus and records all data.

The foamer is a saline solution containing 0.83 wt $\% \mathrm{NaCl}$ (J. T. Baker, reagent grade) with $0.83 \mathrm{wt} \%$ active $\mathrm{C}_{14-16} \alpha$-olefin sulfonate surfactant (Bioterg AS-40, Stepan). Water is provided by a Barnstead Fi Streem II glass still (Barnstead, Thermolyne Corp., Dubuque, lowa). The solution surface tension is 33 $\mathrm{mN} / \mathrm{m}$ measured by the Wilhelmy plate method, and has a viscosity of $1 \mathrm{mPa}$. Bottled bone-dry nitrogen is the gas source.

The core is first flushed with copious amounts (20-100 PV) of $0.83 \mathrm{wt} \%$ brine at $7 \mathrm{MPa}$ backpressure. Periodically, the backpressure is released and then reapplied. This treatment removes virtually all gas and surfactant from the core. Because trace amounts of isopropanol or methanol can have a deleterious effect on foam production, no alcohols are used as foam breakers or as cleaning solvents on any portion of the experimental apparatus. For those experiments where the core is presaturated with aqueous surfactant solution, at least 5 PV of foamer solution is injected to satisfy rock adsorption of surfactant. Measurement of the surfactant elution curve for the core reveals little detectable surfactant adsorption at the surfactant concentration employed. After 1 PV of foamer solution is injected, the concentration of surfactant in the inlet and effluent streams is equal.

In experiments where liquid and gas are injected at constant mass flow rate, the gas/liquid mixture is not foamed before injection. The initial injection rates are not altered until a steady state pressure drop is achieved. After steady state is reached, the liquid and gas rates are varied independently to reach a series of new steady states. In experiments where gas alone is injected at a fixed inlet pressure, the bone-dry nitrogen stream is first passed through a $0.001 \mathrm{~m}^{3}$ (1 liter) stainless steel bomb filled with $0.83 w 1 \%$ brine to saturate the nitrogen with water vapor. In all experiments, the progress of foam propagation is tracked by frequent pressure and saturation sweeps. Discussion of the experimental results is deferred until after review of the foam displacement model description.

\section{Foam Displacement Model}

Previously we outlined a population-balance foam displacement model 15,17 that is easy to implement, fits simply into the framework of current reservoir simulators, employs a minimum number of parameters, and directly embodies pore-level events. This previous work, however, neglected capillary pressure, $\mathrm{P}_{\mathrm{C}}$, in both the flux of gas and liquid and did not explicitly account for the role of capillary pressure in foam-lamellae coalescence. These new effects are included bere.

The first step in formulating the model is to write in standard reservoir simulator form the requisite material balance equations for the gaseous and aqueous phases including the transport and rock adsorption of surfactant 15,17. Including capillary pressure gradients in the transport of gas and liquid is standard (c.f., ref. 18).

The effective resistance of the gas phase is a strong function of foam texture $1-3,12,13$. Therefore, mecharnistic prediction of foam flow in porous media is impossible without a conservation equation accounting for the evolution of foam texture ${ }^{4}$. Following Patzek ${ }^{4}$ and also refs. 1 and 2 we write a transient population balance on the average flowing and trapped bubble size. Rates of accumulation, trapping, convection, generation, and coalescence of foam bubbles are incorporated into a species (i.e., bubble) balance, just as they are for any molecular species in a reservoir simulator.

Mechanistic rate cquations for the generation of foam by capillary snap-off and capillary suction coalescence are also constructed ${ }^{15,17}$. However, we wish now to include capillary-pressure information directly in the expression for foam coalescence. The rate of coalescence is, therefore, written as

$$
r_{c}=k_{1}\left(P_{c}\right) v_{f} n_{f} .
$$

where $v_{f}$ is the local interstitial foam velocity $\left(v_{f}=\right.$ $\left.u f / \phi S_{f}\right), n_{f}$ is the local flowing foam texture (i.e., the number of bubbles per flowing gas volume), and $k_{-}$] the constant for foam coalescence. Equation (1) teaches that a greater flux of lamellae $(v \mathrm{fm} f)$ leads to increased coalescence. $k_{-1}$ reflects the number of foam termination sites. More coalescence sites appear as $P_{\mathcal{C}}$ increases, as shown by the work of Jimenez and Radke ${ }^{19}$. This rate constant is additionally affected by surfactant type and concentration. The thin lamellae pictured in Fig. 1 are stabilized by surfactant adsorbed at the gas-liquid interface. Thus, different surfactant structures achieve varying degrees of stabilization. The ability of a lamella to withstand large capillary pressures before rupturing catastrophically is 
determined by the molecular structure and concentration of the surfactant.

The capillary-pressure dependence, hence saturation dependence, of $k_{-}-1\left(P_{c}\right)$ is quite dramatic. The experiments of Khatib et al. 20 show that for strongly foaming solutions $k_{-}-1\left(P_{c}\right)$ is small for low capillary pressures but rises steeply as $S_{W}$ decreases and $P_{c}$ increases. Since moving lamellae are rapidly stretched under larger $P_{c}$ they become very thin, fragile, and therefore highly vulnerable to breakage. At low aqueous phase saturations sufficient time does not exist for surfactant solution to flow into a rapidly stretched lamella thereby thickening and stabilizing it $^{19}$. In fact, the study of single foam films demonstrates that a characteristic or limiting capillary pressure $\left(P_{c}{ }^{*}\right)$ exists for film breakage 21,22 depending strongly upon surfactant formulation and concentration. When $\mathrm{P}_{c}{ }^{*}$ is met or exceeded foam films spontaneously rupture. Thus, a $P_{c}$ near that corresponding to $\mathrm{P}_{\mathrm{c}}{ }^{*}$ leads to a rate of foam coalescence approaching infinity 20 . Cognizant of these facts we write

$$
k_{-1}\left(P_{c}\right)=k_{-1}^{0}\left(\frac{P_{c}}{P_{c}^{*}-P_{c}}\right)^{2}
$$

where the scaling lactor $\mathrm{k}^{\circ}-1$ is taken as a constant. Equation (2) allows the coalescence rate to increase smoothly from zero at $S_{W}$ equal to 1 to very large values as $P_{c}$ approaches $P_{C}{ }^{*}$. As desired, in the vicinity of the limiting capillary pressure, $P_{c}{ }^{*}$, the slope of the curve given in Eq. (2) approaches infinity.

Because $P_{C}{ }^{*}$ varies with surfactant concentration, another function is necessary for simulations where the porous medium is not presaturated with surfactant. Recent work by Aronson et al. ${ }^{23}$ measured pressure drop in 2.3- $\mu \mathrm{m}^{2}$ beadbacks for $\mathrm{N}_{2}$ foams at a gas fractional flow of about $90 \%$ and also $\mathrm{P}_{\mathrm{C}}{ }^{*}$ for single foam films at a variety of surfactant (sodium dodecyl sulfate) and brine $(\mathrm{NaCl})$ concentrations. This work showed that at elevated brine concentrations (roughly $1 \mathrm{wt} \%$ ) even small concentrations of surfactant $(0.03 \mathrm{wt} \%)$ produced substantial beadpack pressure drops and large rupture pressures for single foam films. The following function for $\mathrm{P}_{\mathcal{C}}{ }^{*}$ at high brine concentrations is suggested by their work

$$
\mathrm{P}_{\mathrm{c}}^{*}=\mathrm{P}_{\mathrm{c}, \max }^{*} \tanh \left(\frac{\mathrm{C}_{\mathrm{s}}}{\mathrm{C}_{\mathrm{s}}^{\mathrm{o}}}\right)
$$

where $\mathrm{P}^{*}$,max is a limiting value for $\mathrm{P}_{\mathrm{C}}{ }^{*}$ and $\mathrm{C}_{\mathrm{s}}^{\mathrm{O}}$ is a reference surfactant concentration for strong net foam generation. We chose values of 0.3 atm (30 $\mathrm{kPa}$ ) and $0.083 \mathrm{wt} \%$ respectively for these two parameters. This function allows $P_{C}{ }^{*}$ to increase rapidly and smoothly from 0 as the surfactant concentration increases and finally to plateau. Hence, $P_{C}{ }^{*}$ is small when $C_{S}$ is small and consequently the rate of coalescence is large and foam cannot form.

\section{Capillary Pressure}

We include capillary pressure via the Leverett Jfunction. The following form of the J-function approximates the capillary pressure relation for our Boise sandstone

$$
J\left(S_{w}\right)=\frac{P_{c}}{\sigma}\left(\frac{\phi}{K}\right)^{1 / 2}=\left(\frac{0.067}{S_{w}-0.15}\right)^{0.2}
$$

where $\phi$ is the rock porosity, $K$ is the absolute permeability, and $\sigma$ is the surface tension of the foamer solution.

\section{Assumntions and parameter fitting}

In the simulatin equations, the aqueous phase is assumed incompressible and nonvolatile, while the gas (i.e., $\mathrm{N}_{2}$ ) is assumed insoluble and ideal. Gravitational effects are neglected. Further, it is assumed that when the core is presaturated with surfactant, the surfactant is present in equal concentration throughout the aqueous phase and that rock adsorption has been satistied. In this instance, the surfactant mass balance is automatically obeyed. If the core is not presaturated with surfactant, we set rock adsorption to zero because the surfactant elution curves 24 for this clean sandstone displayed no significant adsorption loss. Also it is assumed that once foam traps it cannot be displaced. This allows for simulation of so-called continuous-gas foams ${ }^{2}$.

The requisite conservation equations and constitutive relations are incorporated into a standard finite-difference simultaneous solution (SS) simulator with explicit upstream weighting of the phase mobilities and solved (c.f., ref. 18). The four primitive unknowns are pressure, gas-phase saturation, surfactant concentration, and bubble density. Further numerical details are available elsewhere 24 .

Numerical values of parameters for the population balance portion of the model are determined by steady-state measurements. Specifically, steady-state flow trends, saturation, and pressure drop profiles must be matched. Fortunately, this drastically narrows our range of parameter choices. The matching procedure requires only one steady-state pressure profile along with the accompanying steady state trends of pressure drop versus gas velocity at fixed liquid rate and pressure 
drop versus liquid velocity at fixed gas rate. These are easily obtained within one experimental run. For further details of the matching procedure see refs. $15,17,24$. Once the foam displacement parameters are determined, there is no need to make adjustments to accommodate different types of transient injection or initial conditions. Parameter values used here are taken from ref. 15 except for $k^{0}-1$. Since a new function for the foam coalescence rate constant is employed, $k^{0} .1$ is adjusted to $0.017 \mathrm{~cm}^{-1}$.

The solid lines in Figs. 2 and 3 show the results of the steady-state parameter matching procedure described above. Figure 2 reports the steady state system pressure drop versus liquid velocity at constant gas velocity while Fig. 3 displays the steady state pressure drop versus gas velocity (at exit pressure) relationship with liquid velocity held constant at two different levels.

In the steady state we find an excellent fit between experiment and theoretical prediction. In Fig. 2 , the model pressure gradient increases linearly and overlies the experimental data (symbols) almost exactly. In Fig. 3 pressure drop at two different given liquid velocities is shown to be independent of gas velocity. At the liquid velocity of $0.028 \mathrm{~m} /$ day in Fig. 3 (open circles) the foam simulator overpredicts the experimental pressure drop slightly, but matches almost exactly at a liquid velocity of $0.077 \mathrm{~m} /$ day. The overestimation of system pressure gradient is understood by comparing the constant liquid velocity used in Fig. $3(0.028 \mathrm{~m} /$ day $)$ to the results in Fig. 2. The experimental point at $0.028 \mathrm{~m} /$ day on Fig. 2 did not fall on the model predicted line. The data taken during that portion of the current experiment appear to have slightly depressed pressure drops. In general, simulation mimics experiment well in the steadystate mode.

The steady-state pressure-drop trends are a result of the adjustment of foam texture as flow rates change. When gas velocity is varied under constant liquid flow rate conditions, foam texture coarsens, viscosity decreases, and constant pressure drop is maintained. When liquid velocity is increased while gas rates are beld constant, foam texture increases linearly with $v_{w}$ and hence viscosity is adjusted so that Newtonian behavior is found.

\section{Comparison of Theory and Displacement Experiments}

The following four test cases illustrate the efficacy of our population-balance method in reproducing a variety of transient foam-flow behavior. First, we consider two examples of simultaneous injection of gas and surfactant solution at different constant mass injection rates into a core completely saturated with surfactant solution. Nexh, we explore simultaneous injection of gas and surfactant solution, again at constant mass injection rates, into a core that is initially free of surfactant. Lastly, we inject gas at a fixed injection pressure into a surfactant-saturated core. In this case no liquid is injected.

In the transient mode, we wish to determine the length of time required for the system to come to steady state and to verify the existence and track the movement of foam displacement fronts within the porous medium.

Simultaneous Injection inte a Surfactant-Saturated Core

In the first example, injection rates are quite low. Gas is injected at a rate of $0.43 \mathrm{~m} /$ day relative to the exit pressure of $4.8 \mathrm{MPa}$ and foamer solution is injected at $0.046 \mathrm{~m} /$ day into a surfactant saturated core. This yields a quality or gas fractional flow of $90 \%$ at the core exit. Figures 4 and 5 display the model results in addition to the experimental saturation and pressure profiles. Figure 6 displays the foam texture profiles generated by the simulator. The population-balance parameters employed are exactly identical to those above that reproduced steady state foam behavior. Theoretical results are presented as solid lines. Unfortunately, no experimental method currently exists to directly measure foam texture in situ. Dashed lines simply connect the individual data points. Elapsed time is given nondimensionally in pore volumes (PV) which is the ratio of the total volumetric flow rate (at exit pressure) multiplied by elapsed time and divided by the void volume of the core.

Steep saturation fronts are measured and predicted at all time levels (Fig. 4) whereby aqueous-phase saturation upstream of the front is roughly $30 \%$, about 5 units above connate, and downstream it is $100 \%$. Model fronts are somewhat steeper and sharper than those measured experimentally, but the theoretical saturation profiles track experimental results very well. From the saturation profiles it is apparent that foam moves through the core in a piston-like fashion. After the front passes a particular location, saturation changes very little. Even though nitrogen and surfactant solution are injected separately, rapid foam generation and liquid desaturation still occurs very near the core inlet. Gas breakthrough at the core outlet occurs at roughly 1 PV, and little or no change experimentally or theoretically occurs in the saturation profile after breakthrough.

The model further predicts that $S_{w}$ is higher at the core inlet. Aqueous phase saturation is around $40 \%$ at $x / L$ equal to zero, but drops rapidly to approximately $30 \%$ by $x / L$. equal to 0.15 . Since no foam is injected, foam bubble density is essentially zero at the inleh, effective flowing-foam viscosity is equal to the gas viscosity, and, consequently, $S_{W}$ is higher than in the remainder of the core. Including the 
dispersive action of capillary pressure in the material balance fluxes obviates steep gradients in aqueous phase saturation. Without capillary pressure effects in the material balance fluxes, $S_{W}$ is $76 \%$ at the inlet and drops to $30 \%$ by $x / \mathrm{L}$ equal to $0.20^{15,17}$. Minssieux ${ }^{25}$ detected such a region of high $S_{W}$ near the inlet of a sandpack. A region of net foam generation exists near the inlet by implication. Foam texture increases rapidly, but the region where rates of generation and coalescence are out of balance is finite. Unfortunately due to equipment limitations, few experimental data are available in this region.

The region of net foam generation is also witnessed in the transient pressure profiles of Fig. 5 . Both the experimental and model results (solid lines) show that pressure gradients near the inlet are shallow indicating that flow resistance is small. Steep gradients are found downstream of the inlet region. These steep gradients confirm the existence of a strong foam piston-like front moving through the core. In general, large pressure gradients are witnessed where aqueous-phase saturation is low and vice versa. Hence, we infer experimentally that foam texture must be coarse near the inlet and the fraction of foam flowing there large.

These inferences are born out in Fig. 6 which reports model-predicted foam texture as a function of dimensionless distance and time. At all time levels, foam bubbles are coarsely textured near the inlet, but beyond the first fifth. of the core, foam texture becomes nearly constant at each time level. Figure 6 also confirms that foam moves through the column in a piston-like fashion consistent with the experimental data in Figs. 4 and 5 . Further consideration of these three figures shows that the saturation, pressure, and foam texture fronts track exactly both experimentally and theoretically. High pressure gradients and fine foam textures are seen where liquid saturation is low and vice versa.

We notice one more interesting feature of Fig. 6. At times of 0.65 and $0.80 \mathrm{pV}$ the bubble density downstream of the inlet region exceeds the foam texture at steady state. This effect arises because the compressibility of $\mathrm{N}_{2}$ is included. A foam bubble created upstream finds itself out of equilibrium with the local pressure (that is, smaller or more dense than the local pressure demands) when it transports downstream. Hence, the steady-state texture is overshot somewhat as finely textured flowing foam fills the initially liquid-filled regions near the foam front. Coalescence forces coarsen the bubbles over time to the equilibrium density. At steady state, the foam texture decreases away from the inlet region. Essentially, the bubbles expand and hence their number density decreases as they now downstream into lower pressure areas. No overshoot in bubble texture is found in the calculations when the gas phase is made incompressible 24 .
In the second example (Figs. 7 to 9), foam displacement rates are roughly 3 times larger. Gas is injected at $1.2 \mathrm{~m} /$ day relative to the exit pressure of $5.0 \mathrm{MPa}$ and foamer solution is injected at 0.11 $\mathrm{m}$ /day again into a surfactant-saturated core where initially $S_{W}$ is $100 \%$. The gas fractional flow of $92 \%$ is slightly larger than in the first example. It is important to reiterate that model parameters are identical to those used to generate Figs. 2 to 6 . Only the injection rates are changed.

Examination of Figs. 7 and 8 shows that, again, the experimental (symbols and dotted lines) and theoretical (solid lines) transient saturation and pressure profiles agree quite well. Sharp piston-like displacement is evidenced. Because higher rates are used incurring a larger pressure drop across the core and because the gas is compressible, the foam front progresses down the core more slowly than it did in the first example. In Fig. 4 gas breakthrough is somewhat after $0.80 \mathrm{PV}$ while in Fig. 7 it is closer to $1 \mathrm{PV}$. Again steady-state liquid saturation is higher at the core inlet and the pressure gradient shallower than it is farther downstream in the core, because of the region of net foam generation near the beginning of the core. Figure 9 confirms that a piston-like front of foam develops that tracks exactly with the saturation and pressure profiles. The effects of compressibility on bubble texture are even more dramatic in Fig. 9 than they are in Fig. 6.

\section{Simulaneous Iniection inte a Brine-Filled Core}

In Figs. 10 to 13 we inject gas and surfactant solution at fixed mass injection rates into a core completely saturated with brine containing no surfactant. The gas injection rate is $0.43 \mathrm{~m} /$ day while the foamer solution injection rate was $0.077 \mathrm{~m} /$ day to give a gas fractional flow of $85 \%$. The system backpressure was $5.0 \mathrm{MPa}$. Because surfactant is not initially present throughout the core, a slower pressure response than the above two cases is anticipated.

In the transient theoretical saturation profiles shown in Fig. 10 we see that at short times (i.e., $0.10 \mathrm{PV}$ ) two saturation fronts exist. The first front is located at roughly $x / L$ equal to 0.35 and is the distance that unfoamed gas travels into the core. The experimental and theoretical locations of this first front agree well. Little liquid is displaced by this front because gas mobility is bigh in the absence of foam, and, consequently, gas breakthrough is quite rapid when the porous medium is not saturated with surfactant solution. The second front is at approximately $x / L$ equal to 0.06 and is quite steep and sharp. This second saturation front corresponds to the distance surfactant has propagated. Foam forms quite rapidly when surfactant is present. This second saturation front is too close to the core inlet at a time of $0.10 \mathrm{PV}$ to be detected experimentally. 
After gas breakthrough, the foam piston front continues down the core pushing out most of the liquid that the first displacement front left behind. Quite good agreement between simulation and experiment is witnessed even at the later times of 1.0 and 1.6 PV. Foam-front propagation is slow because foam transports only as quickly as surfactant. Foam coalescence is infinite whenever surfactant concentration is zero.

These points are well illustrated on Figs. 11 and 12. Figure 11 presents the transient foam texture history whereas Figure 12 contains the surfactant propagation history. Comparison of these two figures shows that foam texture is quite fine when surfactant concentration is high but falls off dramatically where surfactant concentration is low. In the absence of any surfactant, foam texture is zero. In other words, a continuous channel of unfoamed gas exists.

Figure 13 presents the transient pressure profiles for this case. Because the theoretical saturation profiles track well with experiment, we expect the pressure profiles to track well also. Examination of Fig. 13 shows that this is indeed true. Not only do theoretical and experimental foam-front locations in Fig. 13 match well, but also do the predicted and experimentally determined pressure gradients. Where saturation is low and surfactant concentration bigh, pressure gradients are quite steep and vice versa. As in the earlier cases, the pressure gradients near the core inlet are shallow reflecting the region of net foam generation near the core inlet shown in Fig. 11. The system pressure drop reaches steady state in about 3.5 PV.

\section{Eixed-Pressure Gas Injection}

In the last mode of foam generation, shown in Figs. 14 to 16, gas alone is injected into a surfactantsaturated core such that foam is generated at a fixed pressure drop. Initially the experimental pressure drop established over the core was $380 \mathrm{kPa}(55 \mathrm{psi})$, however as gas discharged from the cylinder, the regulator allowed the pressure to fall to about 300 $\mathrm{kPa}$ (44 psi). The experimental decline in the injection pressure was well documented, thus it was a simple matter to include a declining gas injection pressure into the numerical simulation of this experiment. Figure 14 displays the experimental and simulated pressure profiles. Examination of the system pressure drop at $x / L$ equal to zero shows that the declining injection pressure was indeed accurately modeled. Because of the decline in injection pressure, choice of a gas flow rate for nondimensionalizing time is not clear. Roughly an hour after gas breakthrough, the effluent gas rate on the $0.1 \mathrm{MPa}$ (1 atm) side of the backpressure regulator stabilized at $5.1 \mathrm{~cm}^{3} / \mathrm{s}$ and remained constant. This rate is chosen to nondimensionalize time in both the experiment and simulation. Again the simulation parameters are identical to those used for the three earlier cases.

Figure 15 compares the experimental and simulated saturation profiles. Several aspects of this graph are worthy of note. First, the saturation profiles match moderately well. At times longer than 0.43 PV the predicted front lags somew bat behind the experimental front indicating that the simulator is predicting too efficient of a foam displacement. Thus, the experimental saturation front is moving througn the core more rapidly than is the simulated one. Concomitantly, aqueous-phase saturations upstream of the saturation front do not match as well as they did for the fixed-rate injection schemes. At 0.43 PV the theoretical saturation upstream of the front is slightly above $31 \%$, whereas the actual average saturation is closer to 45\%. After foam breakthrough, the experimental saturations continue to decline slowly as do the simulated ones. Given enough time, the experimental saturations, as well as the simulated ones, must decline to connate saturation, because no liquid is injected. In fact, the simulated profile at 3.8 PV indicates correctly that the core slowly drys out from the front towards the back. The actual experiment was not run long enough that connate liquid saturation and complete collapse of the foam were reached.

Pressure profiles prior to foam breakthrough are shown in Fig. 14 at times of 0.18 and 0.43 PV. Again some discrepancies between the theoretical and actual pressure profiles are seen. In general though the match between the two is acceptable. As suggested by the saturation profiles, the simulated pressure profile at 0.43 PV lags behind the actual profile. Careful study of Fig. 14 shows that the agreement at 3.8 PV is quite good.

When foam is generated with a fixed pressure drop actoss a core, it is customary to also report the effluent gas rate at a variety of time levels to quantify gas production 26,27. For instance, at time levels of $1.1,2.0$, and 3.8 PV the experimental effluent superficial gas velocities are $0.24,0.24$, and 0.25 $\mathrm{cm} / \mathrm{s}$ respectively while the model yields rates of $0.19,0.21$, and $0.21 \mathrm{~cm} / \mathrm{s}$. The difference between experiment and simulation here is consistent with the results discussed in Figs. 14 and 15. The simulator predicts slightly too large of a reduction in gas mobility. This additional manner of comparison also shows that the theoretical model predicts foam behavior adequately.

Further examination of Fig. 14 reveals another interesting feature: the pressure gradients, both from experiment and simulation, are steepest immediately upstream of the foam front. Farther upstream the gradients lessen. The simulated bubble profiles of Fig. 16 explain this behavior. Because only gas is injected and foam generation requires some liquid to be present in order for snap-off and lamellae creation 
to occur, foam texture coarsens rapidly far upstream of the foam front. Due to the reduced availability of liquid, foam genrration cannot keep pace with coalescence, which is quite high because saturation is low and correspondingly the capillary pressure is large. At the displacement front, foam textures are fine (see the profiles at 0.18 and $0.43 \mathrm{PV}$ ) because coalescence has not had time to catch up with generation yet. As the foam piston front moves through the core, foam texture at the front becomes coarser. This is a result of the gas advancing and causing a decline in the pressure drop through the gaseous phase, even though the injection pressure is remaining (fairly) constant. As a consequence, gas velocity and foam generation also decline. The bubble profile at 3.8 PV shows that given enough time, the flowing foam coalesces and the texture declines toward zero. Even if all flowing foam coalesces, a substantial portion of the porous medium contains trapped foam that impedes gas flow.

\section{Conclusions}

We have demonstrated that a foam displacement model based on the bubble population-balance approach well predicts experimental foam displacement under a variety of injection conditions in one-dimension. In general, we find good quantitative agreement between experiment and theory in both the transient and steady states. The numerical values of parameters required for the model are found by fitting steady-state trends and thus are not difficult to obtain. Hence, all simulated results shown here are produced from a single sel of parameters. Because our population-balance formulation is mechanistic, it is general. Thus, extension to large field scales should be possible without parameter adjustment.

Direct incorporation of the role of foam texture into the simulator is the key to its success. Foam texture governs foam flow in porous media. A change in the flow velocity of either wetting liquid or gas must be accommodated by a change in texture and in turn a change in flow resistance. In the transient and steady-state modes, fine foam textures are predicted to lead to large pressure gradients and low liquid saturations, whereas coarse textures lead to lesser gradients and higher liquid saturations.

Specifically, we draw the following conclusions for foam displacement and flow in $1.3 \mu^{2}$ (1.3 D) Boise sandstone at $5 \mathrm{MPa}$ backpressure and for total superficial velocities between 0.40 and $2.1 \mathrm{~m} /$ day.

When gas and liquid are injected simultaneously into an initially aqueous surfactant-solution saturated core the resistance to gas flow builds rapidly in time. Steady state is generally achieved in about 2 PV, and the steady-state aqueous-phase saturation is roughly $30 \%$. The population-balance approach accurately predicts the location of saturation and pressure fronts.
When the porous medium is completely filled with brine but devoid of surfactant, the pressure response is slow. Two displacement fronts emerge. Unfoamed gas moves rapidly through those portions of the core where surfactant is absent. Where surfactant is present, foam forms and the second displacement front builds. The second foam front tracks surfactant propagation through the core. Pressure gradients are large and saturations low where surfactant and foam are present and vice versa. Again the population-balance approach mimics the experimental data.

When gas alone is injected into a core saturated with surfactant solution at a fixed pressure drop, a strong foam displacement front forms rapidly. The flow mobility of gas is reduced by the presence of foam during the displacement and for several PV after gas breakthrough. Although the simulator predicts a slightly larger reduction in gas mobility than is found experimentally, the agreement between the population-balance approach and experiment is quite accoptable.

Finally, we find both experimentally and theoretically that a region of net foam generation exists very close to the inlet face of a linear core. Unfoamed surfactant solution and nitrogen are converted rapidly into a finely textured foam in this region.

\section{Nomenclature}

$\mathrm{C}_{\mathrm{S}} \quad$ surfactant concentration, wt\%

$k$ rate constant, units depend on rate expression

I intensity of gamma-ray beam (counts/sec)

$J$ Leverett J-function

$\mathrm{K}$ permeability, $\mathrm{m}^{2}$

L. length of core, in

nf number density of flowing foam (\# of bubbles/volume of flowing foam)

$\mathrm{p}$ pressure, $\mathrm{Pa}$

PV total pore volumes injected

$P_{c} \quad$ capillary pressure, $p_{n w}-P_{w}, P_{a}$

$r$ foam generation or coalescence rate (\# of bubbles/(time)(volume of gas))

$\mathrm{Si}_{\mathrm{i}} \quad$ phase saturation

ui Darcy velocity of phase $i, \mathrm{~m} / \mathrm{s}$

$v_{i} \quad$ interstitial phase velocity of phase $i, \mathrm{~m} / \mathrm{s}$

$x \quad$ spatial variable, $m$

\section{Greek Lelters}

6 porosity

o surface tension, $\mathrm{N} / \mathrm{m}$

\section{Subscrints}

-1 denotes coalescence rate constant

c coalescenco

d dry

f flowing foam 


\begin{tabular}{|c|c|}
\hline $\mathbf{g}$ & generation \\
\hline $\max$ & maximum \\
\hline nw & nonwetting phase \\
\hline $\mathbf{w}$ & wetting phase \\
\hline \multicolumn{2}{|c|}{ Superscripts } \\
\hline 0 & denotes reference value \\
\hline * & $\begin{array}{l}\text { value corresponds to the limiting capillary } \\
\text { pressure }\end{array}$ \\
\hline
\end{tabular}

\section{Acknowledgement}

This work was supported by the U.S. Department of Energy under Contract No. DE-AC03-76SF00098 to the Lawrence Berkeley Laboratory of the University of California. P. Persoff provided invaluable assistance in setting up the experiments.

\section{References}

1. Friedmann, F, Chen, W. H., and Gauglitz, P. A.: "Experimental and Simulation Study of High-Temperature Foam Displacement in Porous Media," SPERE (February 1991) 37-45.

2. Falls, A. H., Hirasaki, G. J., Pazek, T. W., Gauglitz, P. A., Miller, D. D., and Ratulowski, T.: "Development of a Mechanistic Foam Simulator: The Population Balance and Generation by Snap-Off," SPERE (August 1988) 884-892.

3. Ettinger, R. A.and Radke, C. J: "Influence of Foam Texture on Steady Foam Flow in Berea Sandstone," SPERE (February 1992) 83-90.

4. Patzek, T. W.: "Description of Foam Flow in Porous Media by the Population Balance Method," in Surfactani Based Mohility Centrol Prouress in Miscible-Flood Enhanced Oil Becovery, Smith, D. H. Ed., ACS Symposium Series No. 373, 326-341 (1988).

5. Gillis, J. V.and Radke, C. J.: "A Dual Gas Tracer Technique for Determining Trapped Gas Saturation During Steady Foam Flow in Porous Media," SPE 20519, presented at 65th Annual Technical Conference, New Orleans, LA. September, 1990.

6. Bernard, G. G., Holm, L. W., and Jacobs, W. L.: "Effect of Foam on Trapped Gas Saturation and on Permeability of Porous Media to Water," SPEJ (December 1965) 295-300.

7. Holm, L. W.: "The Mechanisms of Gas and Liquid Flow Through Porous Media in the Presence of Foam," SPEJ (December 1968)
359-369.

8. Huh, D. G.; Handy, L. L.: "Comparison of Steady - and Unsteady-State Flow of Gas and Foaming Solution in Porous Media;" SPERE (February, 1989) 77-84.

9. De Vries, A.S.and Wit, K: "Rheology of Gas/Water Foam in the Quality Range Relevant to Steam Foam," SPERE (May, 1990) 185-92.

10. Friedmann, F.; Jensen, J. A.: "Some Parameters Influencing the Formation and Propagation of Foams in Porous Media," SPE 15087. presented at the SPE Califomia Regional Meeting: Oakland, CA, April, 1986.

11. Sanchez, J. M.; Schechter, R. S.; Monsalve, A. "The Effect of Trace Quantities of Surfactant on Nitrogen/Water Relative Permeabilities;" SPE 15446, presented at the 61st SPE Annunal Technical Conference: New Orleans, LA, October, 1986.

12. Falls, A. H., Muster, J. J., and Ratulowski, J.: "The Apparent Viscosity of Foams in Homogeneous Beadpacks," SPERE (May 1989) 155-164.

13. Hirasaki, G. J. and Lawson: "Mechanisms of Foam Flow in Porous Media: Apparent Viscosity in Smooth Capillaries," SPEJ (April 1985) 176-190.

14. Chambers, K. T. and Radke, C. J.: "Capillary Phenomena in Foam Flow Through Porous Media," in Interfacial Phenomena in Petroleum Recovery, Morrow, N. R. Ed., Marcel Dekker Inc., New York (1991) Ch. 6 191.255.

15. Kovscek, A. R. and Radke, C. J.: "Fundamentals of Foam Transport in Porous Media, " in Eoams in the Petroleum Industry, Schramm, L. L. Ed., ACS Advances in Chemistry Series, to appear 1994.

16. Persoff, P, Radke, C. J., Pruess, K., Benson, S. M., and Witherspoon, P. A.:"A Laboratory Investigation of Foam Flow in Sandstone at Elevated Pressure;" SPERE (August 1991) 365-371.

17. Kovscek, A. R.; Radke, C. J.: "A Comprehensive Description of Transient Foam Flow in Porous Media;" No. FS-9, presented at DOENIPER Field Application of Foams for Oil Production Symposium: Bakersfield, CA, February 1993. 
18. Aziz, K.; Settari, A.: Petroleum Reservoir Simulation, Applied Science Publishers LTD: London, 1979; p125-199.

19. Jiménez, A. I. and Radke, C. J.: "Dynamic Stability of Foam Lamellae Flowing Through a Periodically Constricted Pore," in Oil-Eield. Chemistry: Enhinced Becovery and Production Stimulation, Burchardt, J. K. and Yen,T. F. Eds., ACS Symposium Series No. 396. 460 - 479 (1989).

20. Khatib, Z. I., Hirasaki, G. J., and Falls, A. H.: "Effects of Capillary Pressure on Coalescence and Phase Mobilities in Foams Flowing Through Porous Media," SPERE (August 1988) 919-926.

21. Khristov, K, Krugljakov, P., and Exerowa, D.: "Influence of the Pressure in the Plateau-Gibbs Borders on the Drainage and the Foam," Colloid and Polymer Sci. (1979) 257 506-511.

22. Bergeron, $V$ and Radke, C. J.: "Equilibrium Measurements of Oscillatory Disjoining Pressure," Langmuir (December, 1993) 3020-3026.

23. Aronson, A. S.; Bergeron, V.; Fagan, M. E.; Radke, C.J.: "The Influence of Disjoining Pressure on Foam Stability and Flow in Porous Media," Colloids and Surfaces, to appear.

24. Kovscek, A. R.: PhD thesis, University of California, Berkeley, in preparation (1993).

25. Minnsieux, L.: "Oil Displacement by Foams in Relation to Their Physical Properties in Porous Media," JPT (January, 1974) 100-108.

26. Hanssen, J. E.: "Foam as a Gas-Blocking Agent in Petroleum Reservoirs. I: Emperical Observations and Parametric Study," Journal of Petroleum Science and Engineering, to appear.

27. Hanssen, J. E.: "Foam as a Gas-Blocking Agent in Petroleum Reservoirs. II: Mechanisms of Gas Blockage," Journal of Petroleum Science and Engineering, to appear. 


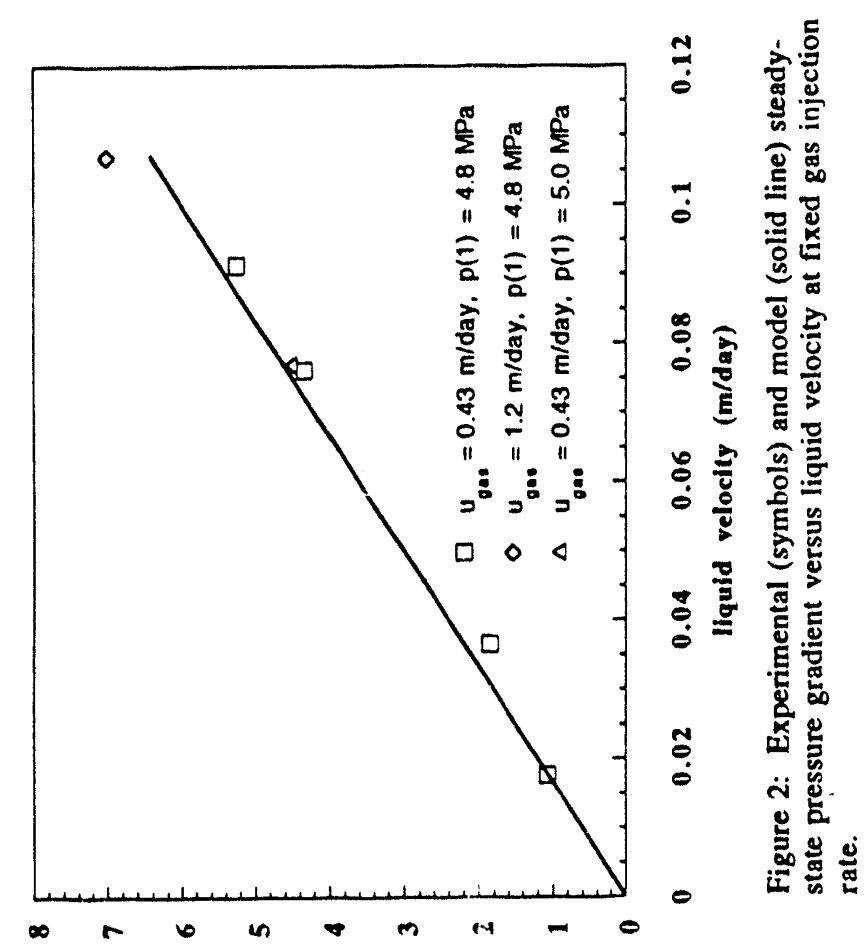

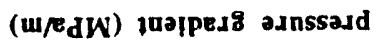

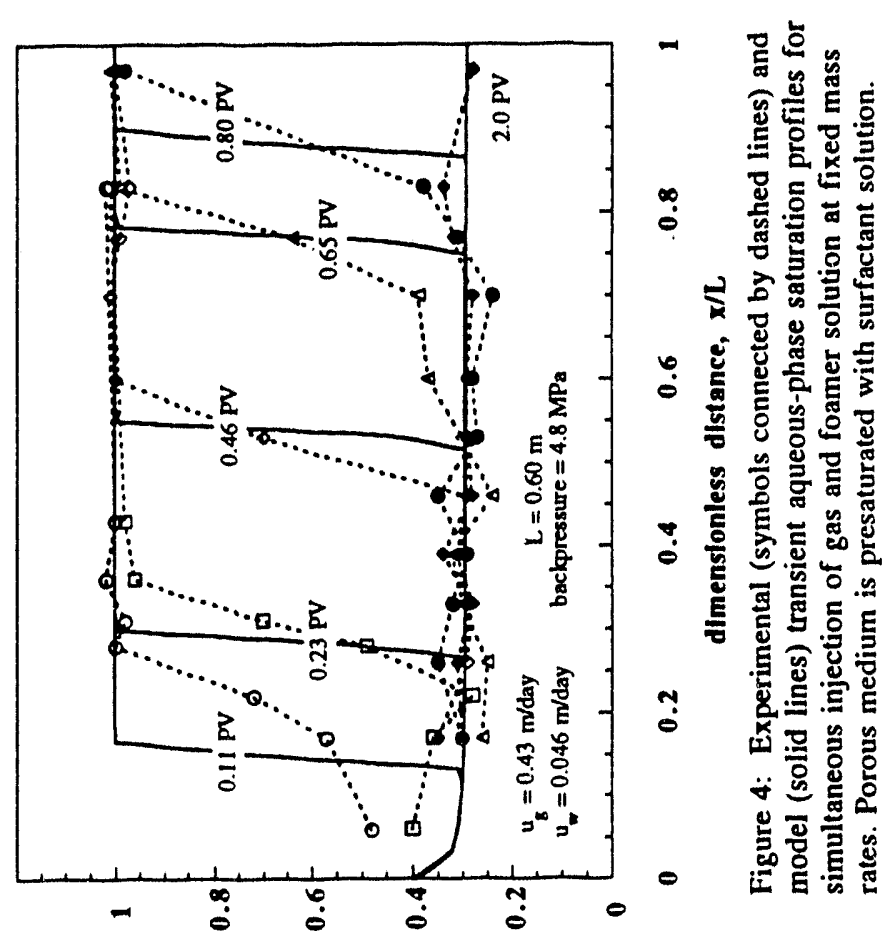

"s 'uollejnjes aseqd snoonbe
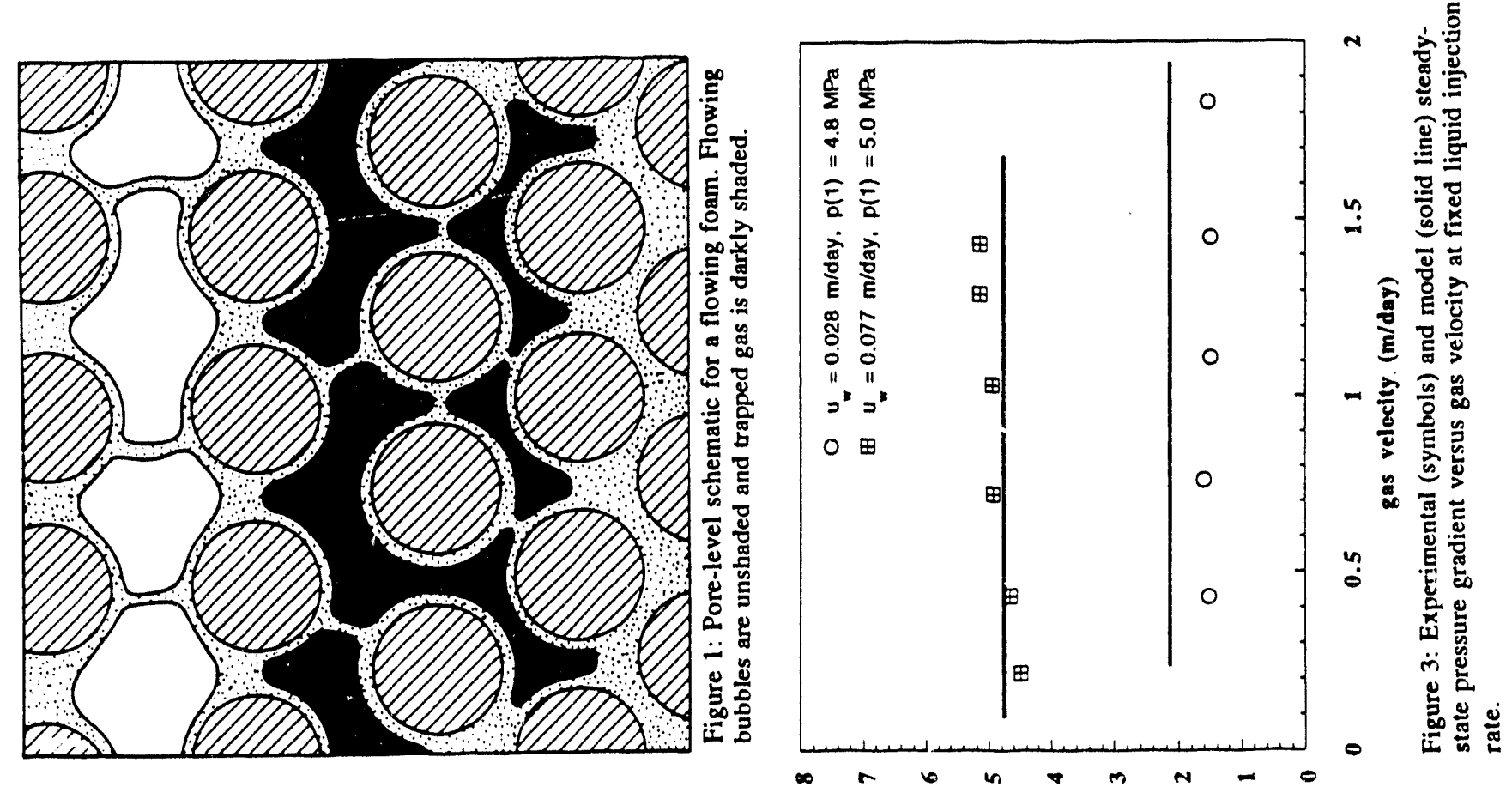

(ui/edW) juappena anssajd 


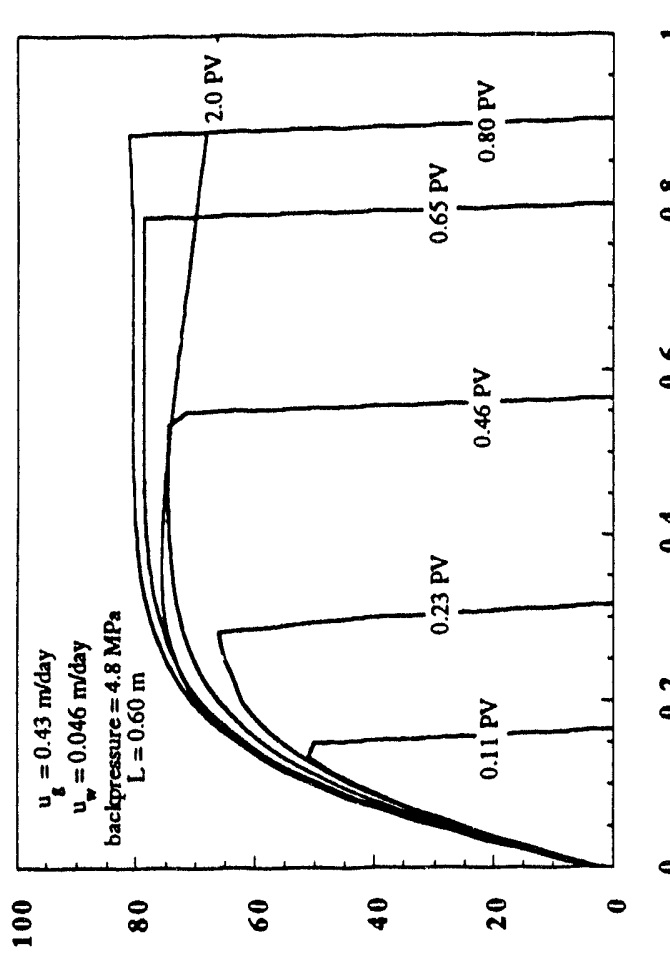

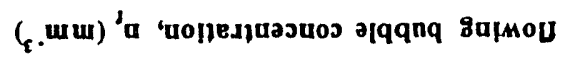
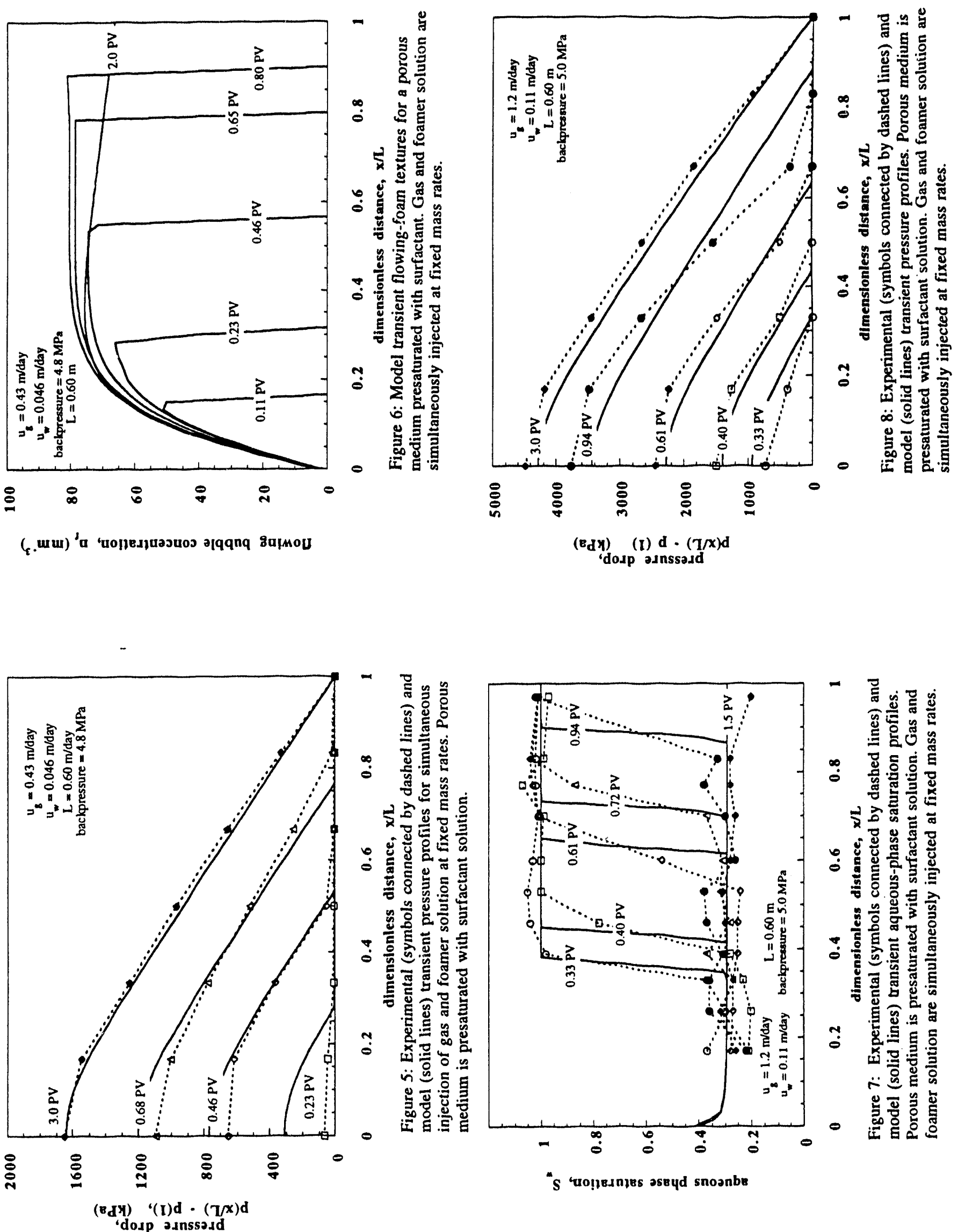

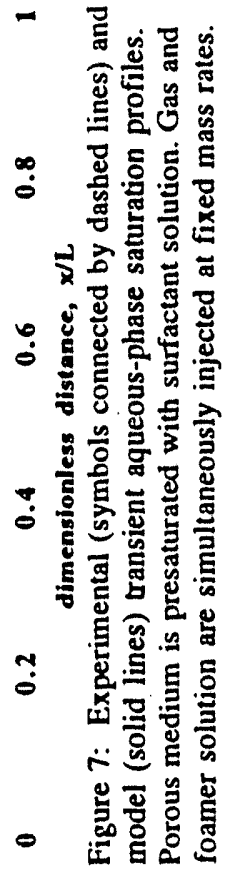

"S uop̣esnies oseyd snoonbs 

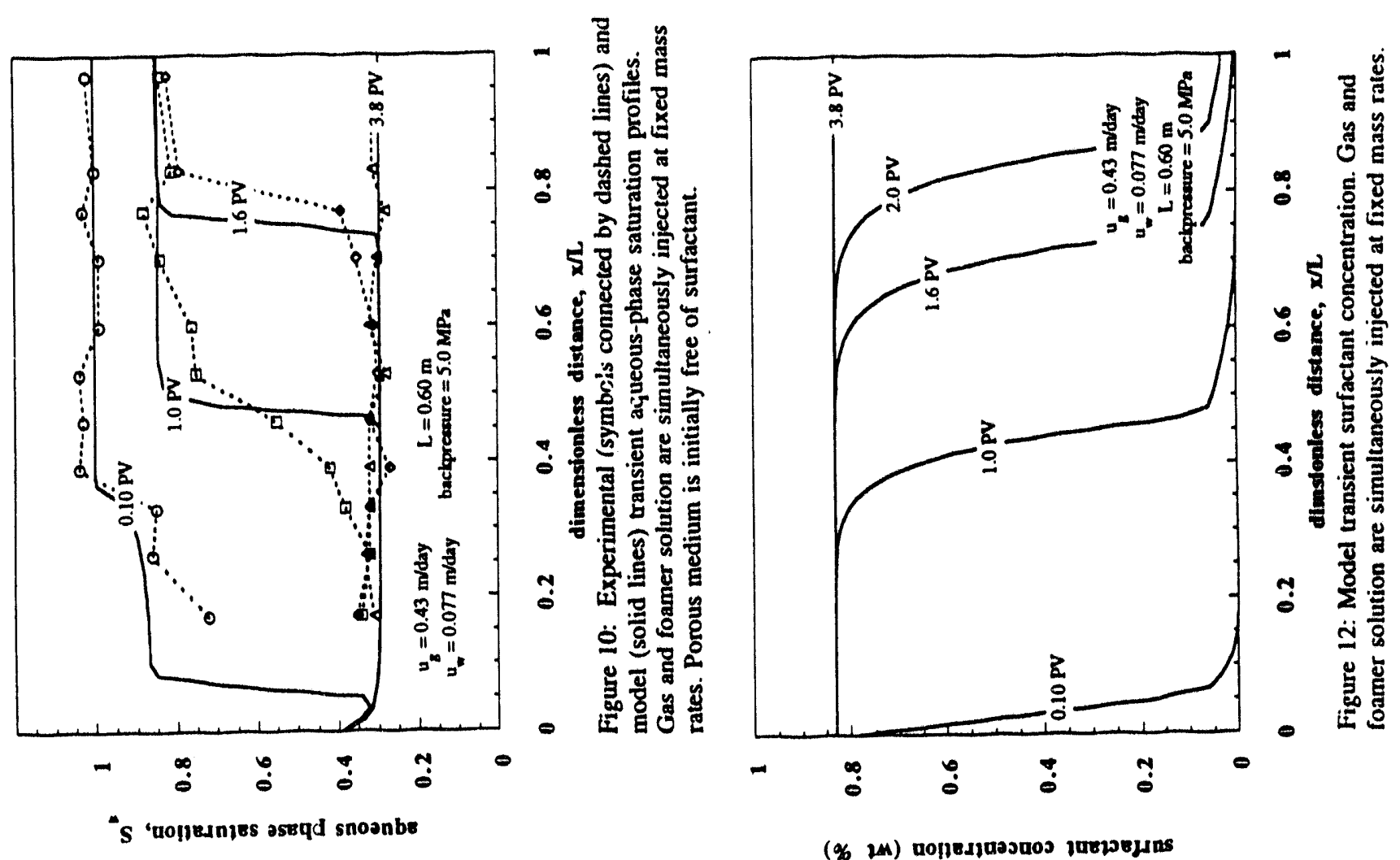

(\% 24) nolpedjuesuos juะ208jus
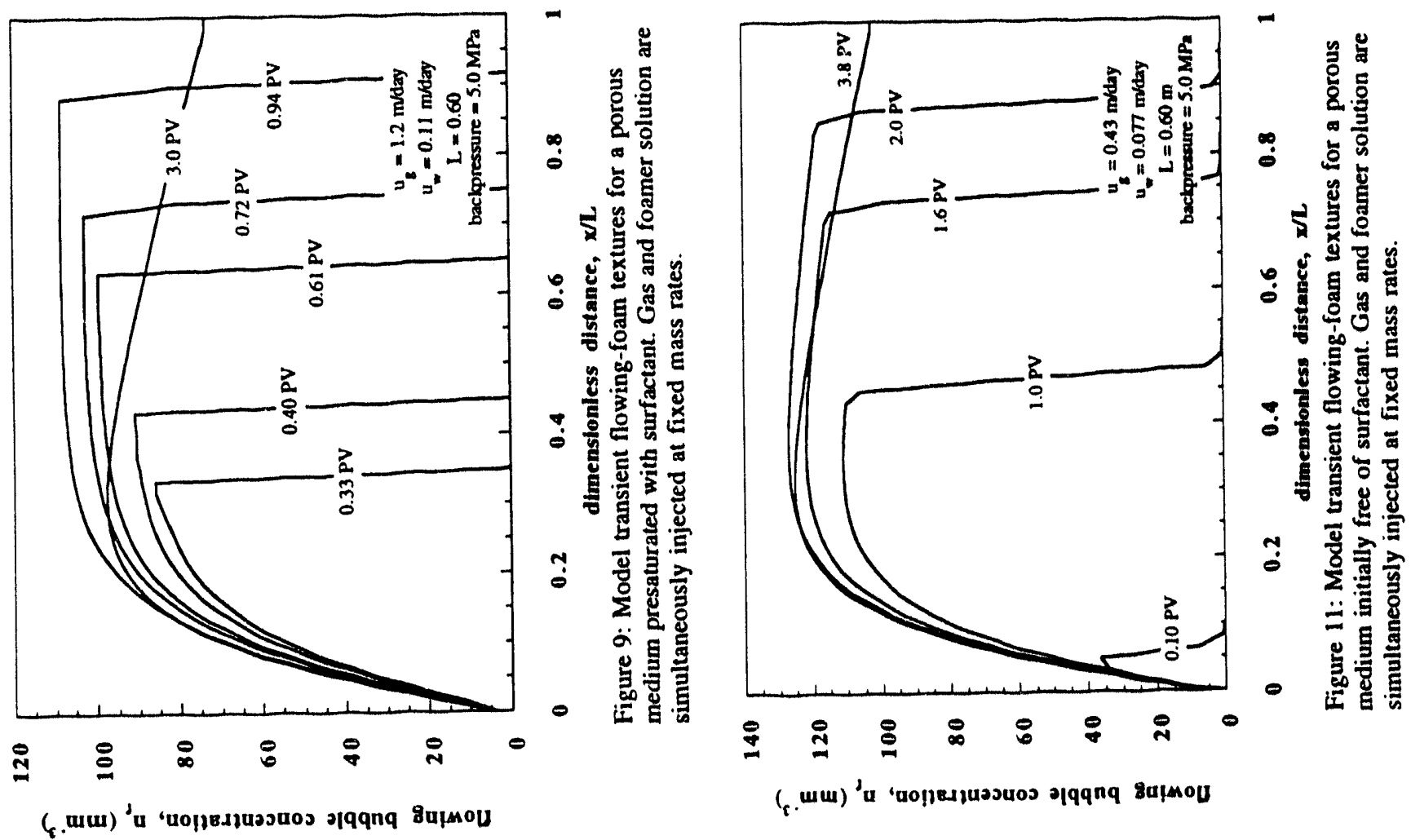

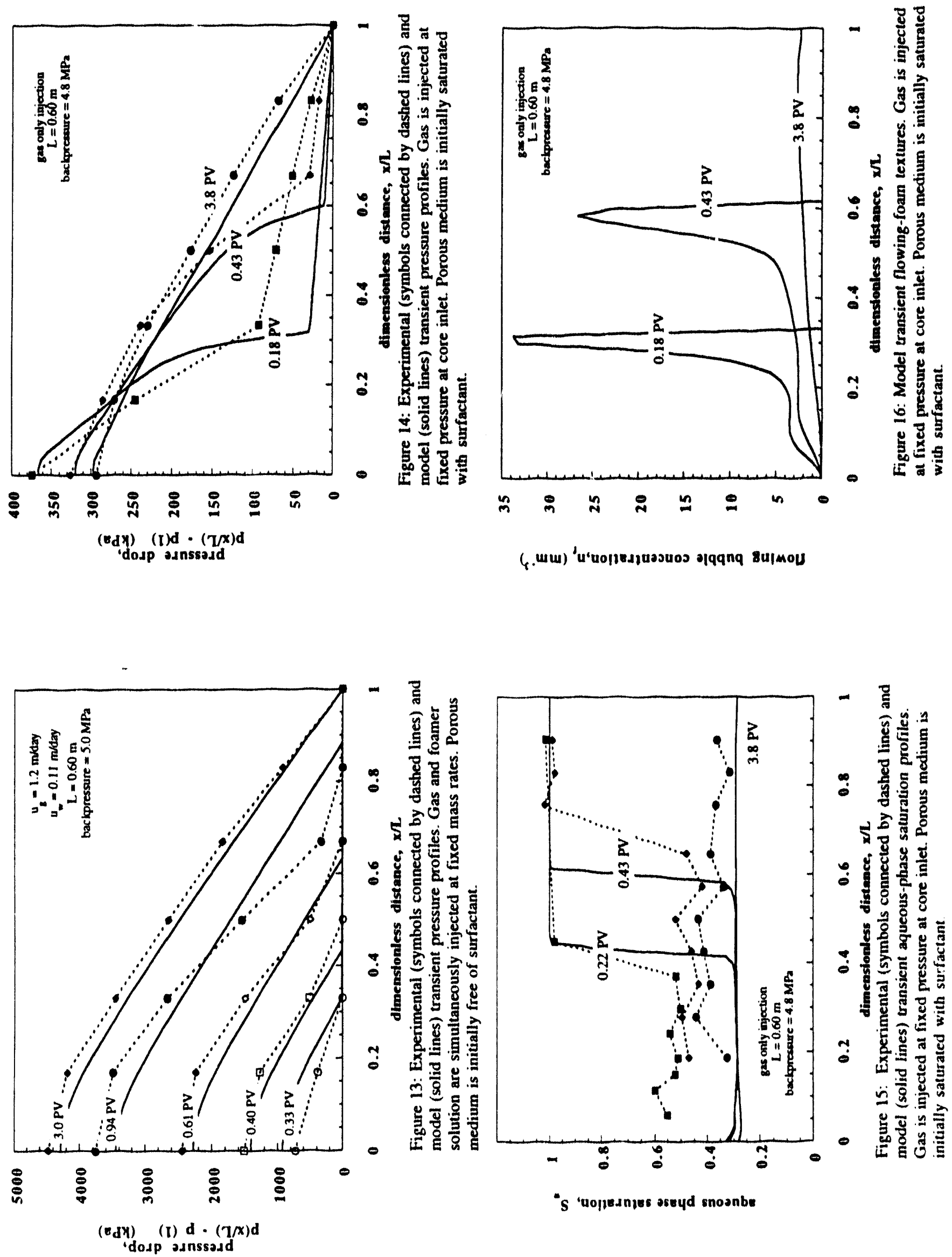

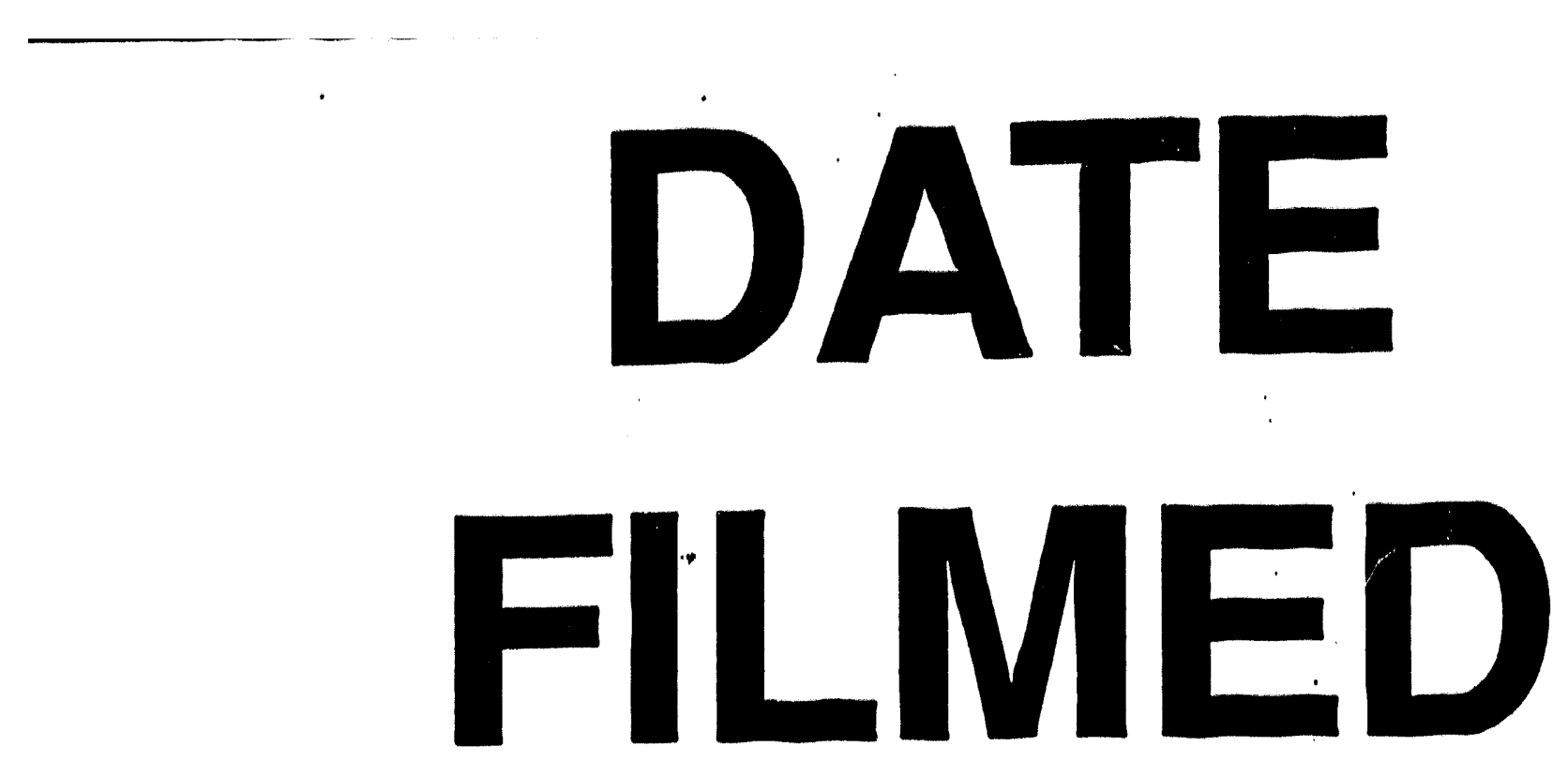

$12 / 13 / 93$
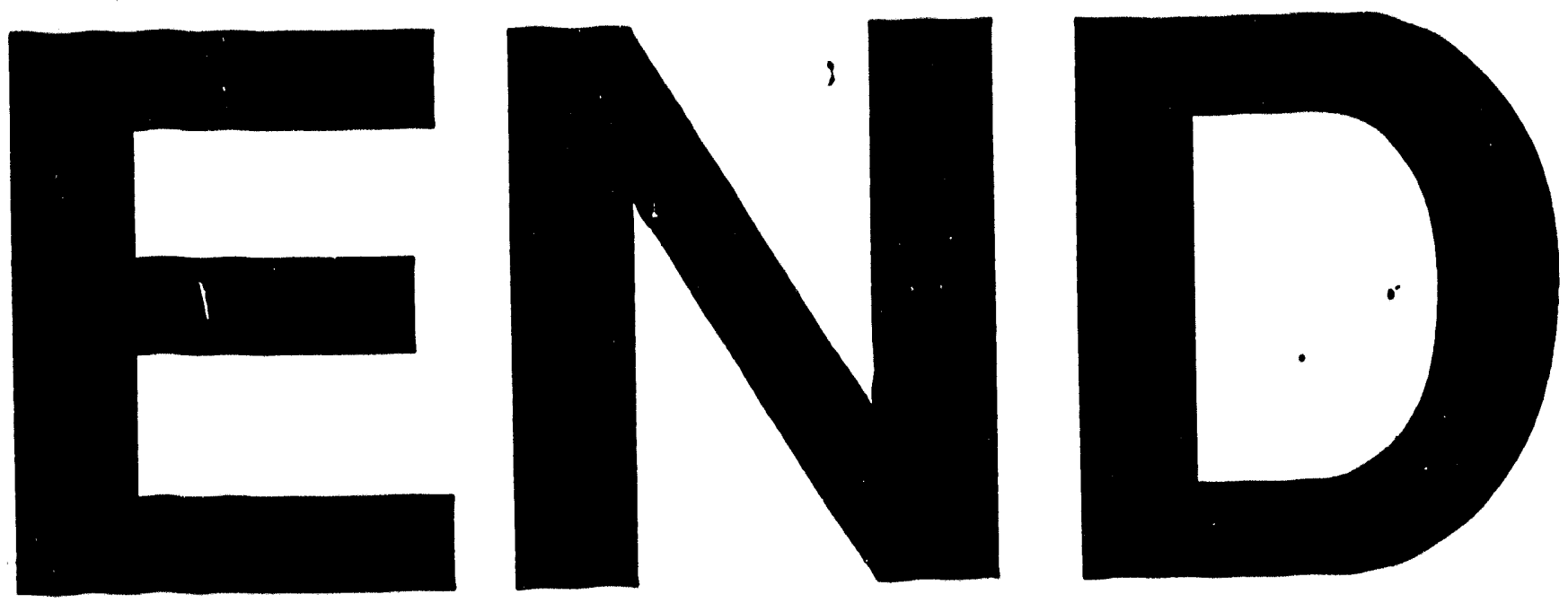
(n) - 1 -

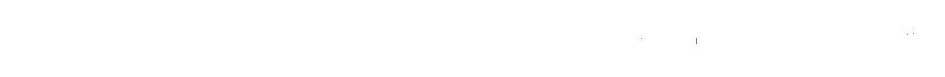

